\title{
EEG-based Upper Alpha Neurofeedback Training Improves Working Memory Performance
}

\author{
Carlos Escolano, Monica Aguilar, Javier Minguez
}

\begin{abstract}
Neurofeedback (NF) training has revealed its therapeutical effects to treat a variety of neurological and psychological disorders, and has demonstrated its feasibility to improve certain cognitive aptitudes in healthy users. Although promising results of $\mathrm{NF}$ training exist in recent literature, the reliability of its effects remains questioned due to a lack of deep studies examining its impact on the human electrophysiology. This paper presents a NF training aimed at improving working memory performance in healthy users by the enhancement of upper alpha band. A user-specific training was used (upper alpha was determined for each user using the individual alpha frequency) to reduce the unspecific factors of training the entire classical alpha band as traditional NF usually does. EEG assessments in active and passive open-eyes state were conducted pre/post the NF training. The EEG analyses reveal the UA enhancement during the active tasks which is independent of other frequency bands. UA was also enhanced in the passive state but independence could not be obtained in lower alpha band. Finally, significant improvement in working memory was obtained with regard to a control group.
\end{abstract}

\section{INTRODUCTION}

The objective of neurofeedback (NF) is to provide the users with operant control of their brain rhythms. Its basic principle consists of measuring the brain activity, decoding or identifying the brain patterns of interest, and then providing the user with feedback stimuli depending on the desired working levels of these rhythms. In NF systems the EEG is the most used recording technique since it is relatively cheap, portable, and has a low set-up cost. Several studies reveal that NF training has therapeutic effects to treat a variety of neurological and psychological disorders such as epilepsy [1], attention deficit hyperactivity disorder (ADHD) [2], and addictive disorders [3], among others. Furthermore, this training applied to healthy users has demonstrated its ability to improve certain cognitive aptitudes [4], [5], [6].

Although behavioral effects of NF training in healthy users have been reported in many works (see [4] for an overview) there is a lack of deep studies examining the effects of NF on the human electrophysiology. In the recent work of Zoefel et al. [6] three main criteria have been proposed to validate a NF training protocol: $(i)$ to assess whether the EEG patterns involved in the NF are trained (trainability); (ii) to asses whether other EEG patterns different from the trained ones are not modified (independence); and (iii) to train a brain

Carlos Escolano and Javier Minguez are with the Instituto de Investigación en Ingeniería de Aragón (I3A) and Dpto. de Informática e Ingeniería de Sistemas (DIIS), Universidad de Zaragoza, Spain. E-mail: \{cescolan, jminguez\}@unizar.es. Monica Aguilar is with Bit\&Brain Technologies S.L, Zaragoza, Spain. E-mail: monicaaguilar@bitbrain.es. This work has been partially supported by projects HYPER-CSD2009-00067, DPI2009-14732C02-01 funded by the Spanish Government. pattern that is hypothesized to be related to a cognitive function in order to increment the probability of obtaining reliable behavioral results (interpretability). The above mentioned work provides solid results of NF by reporting trainability and independence, and cognitive improvement measured in a mental rotation test. In that study the NF training protocol aimed to enhance the upper alpha band.

Alpha rhythm is the dominant frequency in the human EEG. It is characterized by a peak in the range [7.5 - 12.5] $\mathrm{Hz}$ and correlates to several factors such as age, memory performance, brain volume and task demands [7]. Several NF procedures have been applied over the entire classical alpha band (or the SMR band), but failed in fulfilling the three criteria stated above: Vernon et al. found cognitive improvement but changes in the EEG topography rather than a focused increase in the trained frequency bands and scalp locations [4], and Cho et al. showed trainability but cognitive improvement was not assessed [8]. An important problem to achieve those goals in a NF alpha training could be the large inter-user variations presented on alpha frequency, and the unspecific factors of the entire classical alpha band in cognitive performance [7]. Thus, Klimesch et al. [7] introduced the Individual Alpha Frequency (IAF) as a user-specific anchor point that distinguishes between two independent sub-bands of alpha, the upper and the lower ones, since they behave in different ways to certain cognitive demands (upper alpha correlates with cognitive performance).

These findings have been recently applied in the context of NF [5], [6]. Those studies use the IAF to apply NF over the user-specific upper alpha (UA) band to overcome the interuser variations. Hanslmayr et al. study [5] performed NF training in only one day, reporting cognitive improvement in a mental rotation test. Zoefel et al. study [6] was based on the previous work, extending the training over five consecutive days (one week), reporting long-term trainability and independence of UA band, and cognitive enhancement in a mental rotation test.

The present paper complements the NF training procedure proposed in Zoefel et al. [6]. The main objectives of this work are: $(i)$ to evaluate the reliability of the individual upper alpha NF training effects in the EEG (note that reliability is an important problem of neurofeedback); (ii) to explore the hypothesis that the improvement of upper alpha band is associated with an enhancement of the working memory performance [7]; and (iii) to study the effects of individual upper alpha band NF training in EEG acquired in a passive open-eyes resting state. This last objective is relevant in the NF discipline since the EEG recorded in 


\begin{tabular}{|c|c|c|c|c|}
\hline $\begin{array}{c}\text { Passive } \\
\text { (Pre) }\end{array}$ & $\begin{array}{c}\text { Active } \\
\text { (Pre) }\end{array}$ & $\begin{array}{c}\text { NF Training } \\
\text { (5 trials) }\end{array}$ & $\begin{array}{c}\text { Active } \\
\text { (Post) }\end{array}$ & $\begin{array}{c}\text { Passive } \\
\text { (Post) }\end{array}$ \\
\hline $5 \mathrm{~min}$ & $5 \mathrm{~min}$ & $25 \mathrm{~min}=5$ trials $\times 5 \mathrm{~min} /$ trial & $5 \mathrm{~min}$ & $5 \mathrm{~min}$
\end{tabular}

Fig. 1. Session structure. A session was composed by five blocks: a pre/post passive assessment block, a pre/post active assessment block, and the NF training block. Type and duration of each block is shown.

resting condition is the current standard in electrophysiological diagnosis practice. Thus, this evaluation may give insights to better understand the relation of this training to the therapeutic/cognitive improvements reported in the literature.

\section{Methods}

\section{A. Participants}

16 subjects participated in the study. 10 subjects were assigned to the NF training group ( 7 males and 3 females in the range $24.7 \pm 4.11$ years) and 6 to the control group (2 males and 4 females in the range $27.17 \pm 3.97$ years). They had not participated in a NF study before. Participants were duly informed about the entire protocol of the study before signing the consent forms.

\section{B. Signal Recording and Processing}

EEG signals were recorded from 12 active electrodes placed at F3, Fz, F4, C3, Cz, C4, P3, Pz, P4, O1, Oz and O2 (according to the 10/10 system). Ground and reference electrodes were placed on $\mathrm{FPz}$ and the left earlobe, respectively. EOG was recorded from 4 electrodes to detect eyes artifacts: 2 were placed above and below the left eye, and the other 2 in the lateral sides of both eyes. Signal recordings were amplified using a commercial gTec system at a sampling rate of $256 \mathrm{~Hz}$, power-line notch-filtered at $50 \mathrm{~Hz}$, and bandpassfiltered between 0.5 and $60 \mathrm{~Hz}$.

Signal acquisition, processing and feedback presentation were developed using a self-made software written in $\mathrm{C}++$ and running on a Windows computer. Feedback was updated each $30 \mathrm{~ms}$ according to the average of the EEG power in locations $\mathrm{P} 3, \mathrm{Pz}, \mathrm{P} 4, \mathrm{O} 1$ and $\mathrm{O} 2$ (these locations are referred as feedback locations). EEG power was calculated by means of a sliding window FFT algorithm: a 1 second hamming window with $30 \mathrm{~ms}$ of overlapping. Resolution was set to $0.25 \mathrm{~Hz}$ (1024 points using zero-padding). Online artifact detection was performed using a $50 \mu \mathrm{V}$ threshold in the EOG.

\section{Experimental Procedure}

For each subject the experimental procedure consisted of five sessions executed in five consecutive days (one per day, from Monday to Friday). A session was composed of three kind of blocks: $(i)$ passive assessment, $(i i)$ active assessment, and (iii) NF training. Assessment blocks were interleaved pre/post to the NF training block (Figure 1). Complete session lasted 45 minutes for each subject.

a) Passive Assessment: 5-min recording in an openeyes resting state where the subjects were instructed to stay relaxed and focused on a symbol presented in the interface. b) Active Assessment: 5-min recording in an openeyes active state. The pre-block was used to compute the IAF and the baseline for the NF training block. IAF was computed as the peak frequency in the traditional alpha frequency range of $[7.5-12.5] \mathrm{Hz}$ [7]. Upper alpha (UA) was defined as the interval [IAF IAF+2] Hz. Those metrics were recomputed in each session for each feedback location. In both pre/post blocks the subjects faced a square in the interface that changed randomly from gray to red or blue gradually (i.e., changing the saturation). The subjects were instructed to count the number of saturation changes from gray to red in order to challenge them cognitively, thus allowing comparability of the baseline with the NF training.

c) NF Training: 5 trials of 5-min each. In the trials the subjects faced a square in the interface colored either on red or blue when the current average UA power in the feedback locations was greater or lower than the baseline, respectively. The saturation of the square was proportional to the deviation of the average UA power from the baseline. Saturation scale covered $95 \%$ of the power values. Finally, when an artifact was detected the square was colored in gray for 2 seconds.

d) Working Memory Test: A variation of the conceptual span test [9] was used to measure the semantic short-term memory (STM) component in working memory (WM). It was designed a non-clustered version composed of 16 trials. Each trial contained 9 words grouped in 3 different semantic categories. Words were presented at a rate of 1 word per second. After the presentation of 9 words, the subjects had 20 seconds to recall the words. Total test lasted 10 minutes. This test was administered to the NF training group before the first block in the first and last session. Same test was also administered to the control group in the same intervals of time to evaluate the impact of practice effects.

\section{RESULTS}

First, the method used to identify non-responders is described. Non-responders were excluded from posterior analyses. Then, trainability and independence effects of the NF training are studied (for the responders) for the active and passive tasks. Finally, working memory performance is assessed. It must be noted that NF training trials and active assessment blocks will be referred as active tasks in this section, whereas passive assessment blocks will be referred as passive tasks. One participant in the NF group had to be excluded from analysis due to excessive eyes artifacts (no possible reliable estimation of the EEG frequency spectrum).

\section{A. Non-Responders Identification}

Subjects whose average UA power in the last training trial of session 5 was not significantly higher than UA in the pre-active assessment block of session 1 were considered non-responders. As a result, 3 subjects were considered non-responders and were excluded from the analyses (it is interesting to note that those subjects did not show a clear alpha peak in open-eyes passive or active state). Final sample consisted of 6 participants for the NF group. 


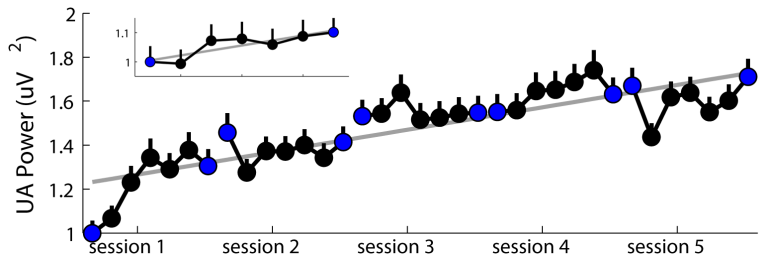

(a) Active tasks: UA trainability

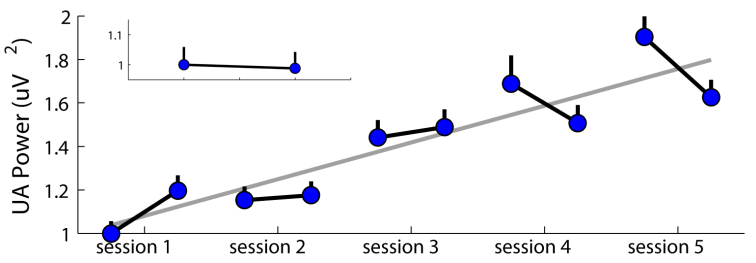

(b) Passive tasks: UA trainability

Fig. 2. (a) UA power across all responders during the temporal course of the study over the active tasks (active assessment blocks and the 5 training trials). (b) UA power over the passive tasks (passive assessments). In the upper corner the average UA progress within sessions is depicted. Blue points denote pre/post active blocks and passive blocks in figures A and B, respectively. Black points denote training trials. Grey line shows the tendency. Values are normalized for each subject to the UA power measured in the first block of the first session. The vertical bars indicate standard errors of the mean.

\section{B. Trainability}

In order to evaluate trainability, the average UA power was computed for all the blocks of each session and all responder subjects. These measures were grouped into the active and the passive tasks (Figure 2). Statistical significance has been assessed using paired, one-tailed t-tests.

Trainability in the active tasks is reflected by a significantly positive tendency of the UA power across the sessions (Figure 2A): the gradients of a fitted regression line for each subject were significantly larger than zero $(t(5)=2.74, p=$ $0.02)$. Furthermore, the UA power during the pre-active assessment of session 5 showed a significantly higher power than the analog block of session $1(t(5)=3.87, p=0.0059)$. The absolute UA power for the pre/post active assessments averaged for all the sessions was $11.76 \mu \mathrm{V}^{2}$ and $12.73 \mu \mathrm{V}^{2}$, respectively; indicating a positive UA power tendency within a session (non significant). The effects of the NF training in a passive resting state are also displayed (Figure 2B). A significant positive tendency of the UA power across sessions was obtained: the gradients of a fitted regression line for each subject were significantly larger than zero $(t(5)=2.76, p=$ $0.019)$. Furthermore, the UA power during the pre-passive assessment of session 5 showed a significantly higher power than the analog block of session $1(t(5)=3.79, p=0.0063)$. The absolute UA power for the pre/post passive assessments averaged for all the sessions was $13.59 \mu V^{2}$ and $14.1 \mu V^{2}$, respectively; indicating a positive UA power tendency within a session (non significant).

The obtained results in the active tasks are in line with those obtained by Zoefel et al. [6]. A linear increase is seen, with the training effects remaining present over sessions. Each training session is built upon the previous one even before training (pre-active block power of each session was higher than the post-active block of previous session). In addition, this training causes a linear increase in the resting EEG. Each session is built upon the previous one but more variability is visible, which might be due to the execution of a more uncontrolled task (relax). Pre/post passive power decrease in sessions 4 and 5 might reflect a saturation effect. When comparing the pre/post passive absolute UA power with the pre/post active power in assessments the passive ones are higher, which might be explained because active assessments involved a cognitive-demanding task, leading to a more pronounced desynchronization (decrease) [7].

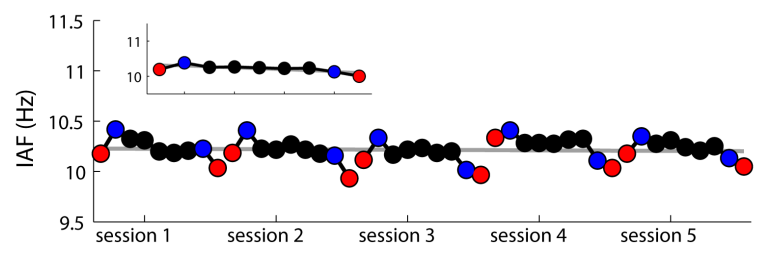

Fig. 3. IAF obtained across the sessions for the responder subjects. Red points denote pre/post passive assessment blocks, blue ones pre/post active assessment blocks, and black ones training trials. Grey line shows the tendency. In the upper corner the average IAF within sessions is depicted.

The IAF remained constant across the sessions (Figure $3)$ : the gradients of a fitted regression line for each subject were not significantly larger than zero $(t(5)=0.22, p=$ $0.83)$. However, the IAF decreased significantly within a session $(t(5)=2.32, p=0.034)$. The average IAF for the pre/post active assessments for all the sessions was 10.38 $\mathrm{Hz}$ and $10.12 \mathrm{~Hz}$, respectively. The analog value for the passive assessments was $10.19 \mathrm{~Hz}$ and $10 \mathrm{~Hz}$, respectively. The increment of IAF in the active assessment with regard to the passive one might be due to the execution of a more cognitive-demanding task, whereas the decrease of IAF within a session might be caused by NF training fatigue [10].

\section{Independence}

Since NF training has focused on the upper alpha band [IAF IAF+2] independence is assessed in the frequency bands close to UA as stated in Zoefel et al. study [6]: (i) lower alpha [IAF-3 IAF-1], and (ii) lower beta [IAF+3 IAF+5]. Statistical significance has been assessed using paired, one-tailed t-tests.

The independence of the EEG frequency spectrum was assessed in the active and passive tasks (Figure 4). In the active tasks, independence was measured between the preactive assessment block of the first and the last session. Upper alpha enhancement ratio (0.97), which can be visually seen as the area below the dashed line in Figure 4, was higher than the lower alpha and the lower beta ones $(0.35$ and 0.087, respectively). Significant differences were found in UA band $(t(5)=3.43, p=0.0094)$, whereas no significant differences were found in lower beta and lower alpha. These results are in line with those reported by Zoefel et al. [6] where independence was also found with regard to the same bands while performing active tasks. 


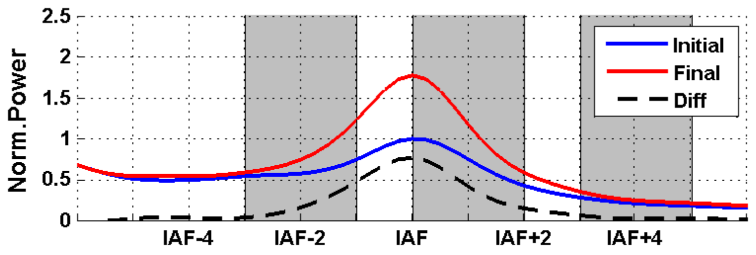

(a) Active state

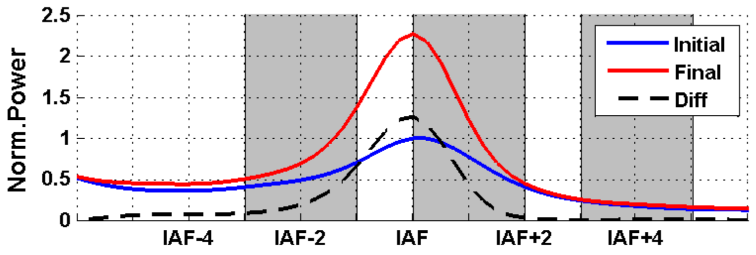

(b) Passive state

Fig. 4. Frequency spectrum in the range [IAF-6 IAF+6] for the active (a) and passive state (b). In (a) the initial state is the pre-active assessment block in session 1 and final state is the analog block of session 5, in (b) the initial state is the pre-passive assessment block in session 1 and final state is the analog block of session 5. Dashed line shows the difference between the final and the initial state, thus indicating the enhancement ratio. The frequency bands where independence is assessed are shaded grey. It must be noted that in order to allow comparability among subjects, EEG power for each bin has been normalized to the IAF power of the initial state.

Regarding the passive tasks, independence was measured between the pre-passive block of the first and the last session. Upper alpha enhancement ratio (1.25) was higher than the lower alpha and the lower beta ones $(0.45$ and 0.039 , respectively). Significant differences were found in UA $(t(5)=$ $2.48, p=0.028)$. In this case significant differences were also found in the lower alpha band $(t(5)=2.45, p=0.029)$. Thus, independence could not be confirmed in a passive resting state with regard to lower alpha band.

\section{Memory Performance}

The scores of the conceptual span test are shown in Figure 5. It can be seen as the NF group has a distribution with higher scores and does not overlap with the 25th and 75th percentiles of the control distribution. The mean increase was $1 \pm 5.83$ words for the control group, and $10.83 \pm$ 7.67 words for the NF group. A paired, one-tailed t-test for independent samples was used to assess significance, obtaining a significant increase for the NF group $(t(9)=$ $2.34, p=0.022)$. EEG was not recorded for the control group, but one could assume that significant changes did not happen in UA power between day 1 and day 5. In summary, NF training group showed a significant enhancement of UA and performed significantly better in a working memory test, thus suggesting a cognitive improvement due to the NF training procedure.

\section{Conclusions}

This paper has presented a NF training procedure of the individual upper alpha frequency band to improve working memory performance in healthy subjects. This works extends the study of Zoefel et al. [6] by assessing the users EEG in a passive resting state to evaluate the effects of NF training when no cognitive effort is being exerted. The results show the trainability and independence of the upper alpha band during the active tasks, thus confirming the reliability of the upper alpha training effects in the EEG. In addition, the effects of the NF training on the electrophysiology of resting EEG reveals an enhancement of upper alpha but no independence could be obtained with regard to lower alpha band. Finally, this NF training was hypothesized to improve working memory performance [7] and it was confirmed: a significant enhancement in memory performance was obtained with regard to a control group.

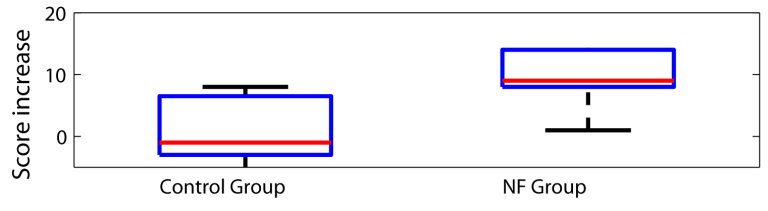

Fig. 5. Increase in the working memory test score (final minus initial scores) for the control and the NF training group. On each box, the central mark is the median, the edges of the box are the 25 th and 75 th percentiles.

Authors are currently investigating predictors of NF training success. Furthermore, more experiments are being carried out to further explore the benefits of upper alpha enhancement in cognitive performance.

\section{REFERENCES}

[1] M.B. Sterman, "Basic concepts and clinical findings in the treatment of seizure disorders with EEG operant conditioning," Clinical Electroencephalography, vol. 31, no. 1, pp. $45-55,2000$.

[2] V.J. Monastra, S. Lynn, M. Linden, J.F. Lubar, J. Gruzelier, and T.J. LaVaque, "Electroencephalographic biofeedback in the treatment of attention-deficit/hyperactivity disorder," Applied Psychophysiology and Biofeedback, vol. 30, pp. 95-114, 2005.

[3] E.G. Peniston and P.J. Kulkosky, "Alpha-theta brainwave training and beta-endorphin levels in alcoholics," Alcoholism: Clinical and Experimental Research, vol. 13, no. 2, pp. 271-279, 1989.

[4] D.J. Vernon, "Can neurofeedback training enhance performance? An evaluation of the evidence with implications for future research," Applied Psychophysiology and Biofeedback, vol. 30, pp. 347-364, 2005.

[5] S. Hanslmayr, P. Sauseng, M. Doppelmayr, M. Schabus, and W. Klimesch, "Increasing individual upper alpha power by neurofeedback improves cognitive performance in human subjects," Applied Psychophysiology and Biofeedback, vol. 30, pp. 1-10, 2005.

[6] B. Zoefel, R.J. Huster, and C.S. Herrmann, "Neurofeedback training of the upper alpha frequency band in EEG improves cognitive performance," Neurolmage, vol. 54, no. 2, pp. 1427 - 1431, 2011.

[7] W. Klimesch, "EEG alpha and theta oscillations reflect cognitive and memory performance: a review and analysis," Brain Research Reviews, vol. 29, no. 2-3, pp. 169 - 195, 1999.

[8] M.K. Cho, H.S. Jang, S.H. Jeong, I.S. Jang, B.J. Choi, and M.G. Lee, "Alpha neurofeedback improves the maintaining ability of alpha activity," NeuroReport, vol. 19, pp. 315 - 317, 2008.

[9] H.J. Haarmann, E.J. Davelaar, and M. Usher, "Individual differences in semantic short-term memory capacity and reading comprehension," Journal of Memory and Language, vol. 48, no. 2, pp. 320 - 345, 2003.

[10] E. Angelakis, J.F. Lubar, S. Stathopoulou, and J. Kounios, "Peak alpha frequency: an electroencephalographic measure of cognitive preparedness," Clinical Neurophysiology, vol. 115, no. 4, pp. 887 - 897, 2004. 